\title{
Configuração e organização de imagens de serviços: um estudo exploratório
}

\author{
Gabriel Sperandio Milan \\ LOPP/PPGEP - Escola de Engenharia da UFRGS \\ Deonir De Toni \\ PPGA - Escola de Administração da UFRGS \\ LISANDRA BARAZETTI \\ Psicóloga Organizacional
}

\begin{abstract}
Resumo
O artigo aborda o desenvolvimento de uma pesquisa exploratória sobre a configuração de imagens no ambiente de serviços. Como ambiente de pesquisa, foi utilizado o Setor de Fisioterapia de um Plano de Saúde, no qual foi possível identificar três tipos de paciente com características específicas (pós-operatórios, neurológicos e crônicos). Com base na Teoria do Núcleo Central, foram identificadas as imagens dos serviços de fisioterapia para cada tipo de paciente, relacionando-as a determinados atributos. Tais atributos foram relacionados a um conjunto de elementos que constituem as imagens, o qual é composto por elementos funcionais, simbólicos, cognitivos e emocionais. Finalmente, no intuito de verificar e analisar o desempenho dos serviços prestados, sob a perspectiva de seus usuários, foi aplicada uma Escala de Diferencial Semântico e uma questão relativa ao grau de satisfação geral, o que pode consolidar ou redirecionar o processo de tomada de decisões.
\end{abstract}

\section{Palavras-chave}

Imagens de serviços, configuração de imagens, organização de imagens, qualidade em serviços, serviços de fisioterapia.

\section{Configuration and organization of the images of services: an exploratory study}

\begin{abstract}
The paper covers the development of an exploratory research about the image configuration in the service environment. To make this project, it was used the Sector of Physiotherapy of a Health Plan, in which it was possible to identify three kinds of patients with specific characteristics (pos-operator, neurological and chronic). With base on the Central Nucleus Theory, it was identified the images of the physiotherapy services for each kind of patient, relating them to determinate attribute. These attributes were related to some elements that make the images. These elements are: functional, symbolic, cognitive and emotional. Finally, with the objective to check and analyze the performance of the service done, under a perspective of the users, it was applied a Scale of Semantic Differential and a question about the overall satisfaction, what can help the manager in the process of making decisions.
\end{abstract}

Key words

Images of services, images configuration, images organization, services quality, physiotherapy services. 


\section{INTRODUĈ̣̃O}

As pesquisas sobre imagens têm focado principalmente imagens relacionadas a lojas, corporações e marcas (DOBNI; ZINKHAN, 1990; BARICH; KOTLER, 1991; STERN et al., 2001). Assim, estudos direcionados ao entendimento de imagens de serviços são oportunos. A partir da questão de pesquisa: "como configurar as imagens de serviço?", foram estabelecidos os seguintes objetivos: identificar as imagens de serviços na percepção de seus usuários, considerando os três tipos de paciente dos serviços de fisioterapia (pós-operatórios, neurológicos e crônicos) de um Plano de Saúde, analisando, também, a composição das imagens no que se refere ao seu conjunto de elementos (funcionais, simbólicos, cognitivos e emocionais); verificar a organização interna dos atributos relacionados à imagem dos serviços; e avaliar alguns aspectos atinentes ao desempenho do provedor de serviços.

Como uma construção sistêmica, as imagens de serviços podem ser configuradas a partir de um conjunto de elementos funcionais, simbólicos, cognitivos e emocionais, revelando como as imagens podem ser configuradas e quais são os elementos mais relevantes para os clientes. A compreensão das imagens, sua organização quanto aos sistemas centrais ou periféricos e os elementos constituintes, torna-se um importante trunfo para os profissionais ligados à gestão das organizações, qualificando o direcionamento de suas ações estratégicas.

Tendo em vista a natureza dos serviços, o mapeamento de suas imagens exige a adoção de técnicas mais específicas e adequadas ao estudo. Por isso, este trabalho apresenta um método muito utilizado na psicologia social, o qual parte da Teoria do Núcleo Central, e que visa, fundamentalmente, identificar as imagens e sua organização no que diz respeito à percepção dos pesquisados. Além deste método, o trabalho emprega uma Escala de Diferencial Semântico, uma técnica muito utilizada na área de marketing, constituindo-se em um instrumento útil para a mensuração de imagens de serviços e uma questão sobre a satisfação geral dos respondentes.

A primeira parte do trabalho apresenta uma revisão teórica sobre a evolução dos serviços na economia, suas características e sobre a qualidade percebida. Na sequiência, são resgatados os diferentes conceitos de imagem, a forma como estas podem ser organizadas e os elementos constituintes das imagens de serviços. Na segunda parte, o método de pesquisa é descrito e, finalmente, na terceira parte, os resultados da pesquisa são apresentados, encaminhando-se algumas considerações finais.

\section{A EVOLUC̣ÃO DOS SERVIC̣OS NA ECONOMIA}

O setor de serviços foi escolhido como ambiência para o desenvolvimento deste trabalho, basicamente, por três motivos: pelo fato da literatura sobre imagens de serviços ser ainda muito incipiente, pela sua representatividade na economia mundial e pela importância na sociedade. Por isso, é relevante que se resgate a evolução dos serviços na economia.

Em 1900, cerca de 30\% da força de trabalho nos Estados Unidos estava empregada no setor de serviços (BATESON; HOFFMAN, 2001) e, em 1929, representava $55 \%$ da população economicamente ativa, sendo que, em $1948,54 \%$ do PIB (Produto Interno Bruto) americano era proveniente do setor terciário (ZEITHAML; BITNER, 2000). Dados publicados em 1984 pelo GATT - General Agreement on Tariffs and Trade (Acordo Geral de Tarifas e Comércio) indicavam que o setor terciário da economia respondia por $66 \%$ da economia norte-americana e por 58\% do Mercado Comum Europeu.

Demonstrando uma tendência ascendente em sua representatividade na economia mundial, em 1990, o setor respondia por 58\% do PIB no Japão e $60 \%$ na União Européia (BATESON; HOFFMAN, 2001). Em 1996, os serviços eram responsáveis por $76 \%$ do PIB norteamericano (ZEITHAML; BITNER, 2000) e, atualmente, no Canadá, respondem por $67 \%$ da geração de riquezas (LOVELOCK; WRIGHT, 2001).

\section{A s imagens de serviços podem ser A configuradas a partir de um conjunto de elementos funcionais, simbólicos, emocionais e cognitivos.}

$\mathrm{Na}$ economia norte-americana, caracterizada por sua liderança mundial, entre as décadas de 80 e 90, havia, no setor de serviços, um índice de empregabilidade de $70 \%$ em relação à população economicamente ativa (ALBRECHT; ZEMKE, 1990), enquanto que, atualmente, $79 \%$ das pessoas empregadas estão inseridas em atividades de serviços (ZEITHAML; BITNER, 2000). Um estudo desenvolvido pelo Bureau of Labor Statistics identifica que há uma forte expectativa de que as ocupações advindas dos serviços serão responsáveis por todo o crescimento no número de empregos até o ano de 2005 (BATESON; HOFFMAN, 2001). No Brasil, de acordo com dados do IBGE (Instituto Brasileiro de Geografia e Estatística), em 1998, os serviços respondiam por 
$59,70 \%$ da economia brasileira, seguidos pela indústria, com $32,30 \%$, e a agricultura, com $8,00 \%$ (BUSINESS MONITOR INTERNATIONAL, 2000), dados estes que vêm se mantendo.

\section{CARACTERÍSTICAS DOS SERVICOS E A QUALIDADE PERCEBIDA}

Os serviços apresentam uma natureza diferenciada em comparação aos produtos, tendo em vista a incidência de quatro características: intangibilidade, os serviços não podem ser tocados; perecibilidade ou deteriorabilidade, os serviços não podem ser estocados; simultaneidade ou inseparabilidade, os serviços são produzidos e consumidos ao mesmo tempo; e heterogeneidade ou variabilidade, potencial de alta variabilidade no desempenho dos serviços (BERRY, 1980; ZEITHAML et al., 1985; GRÖNROOS, 1990; dentre outros). Por isso, é significativo tanto para o meio acadêmico quanto para os gestores de empresas de serviços aprofundarem estudos sobre o setor.

Por outro lado, ao abordar a qualidade, observa-se pouca convergência a respeito de sua definição (HUFF et al., 1994). Como exemplo disso, pode-se citar algumas definições de qualidade, as quais abordam diferentes enfoques. Qualidade é conformação a especificações (CROSBY, 1979), melhoria da conformação a especificações através da redução da incerteza e variabilidade (DEMING, 1986), adequação ao uso (JURAN, 1989) e o julgamento do consumidor sobre a excelência global ou superioridade de um produto (ZEITHAML, 1988).

No entanto, Grönroos (1990) e Fornell (1991) enfatizam que o que realmente interessa é a definição do que seja qualidade sob a ótica do cliente. Portanto, qualidade é conformação às especificações do cliente, e o que conta é o que o cliente percebe como qualidade. Ao avaliar o desempenho de um produto ou serviço, o cliente geralmente analisa seus principais atributos (OLIVER, 1997). Neste sentido, a mensuração do nível de satisfação dos clientes serve como uma medida de verificação do desempenho global da empresa em relação às expectativas dos clientes (ANDERSON et al., 1992).

Enquanto os economistas têm se interessado pela aferição da satisfação de clientes como uma representação da avaliação da qualidade, os psicólogos têm se importado com as expectativas e percepções dos clientes como antecedentes da satisfação (JOHNSON; FORNELL, 1991). O risco das empresas, ao interpretarem a satisfação dos clientes tomando por base a qualidade, está no fato de que os aspectos atinentes à qualidade são determinados, normalmente, pela percepção dos gerentes, porém, em muitos casos, tal entendimento pode diferir da visão dos clientes (ZEITHAML et al., 1988).
Assim, a qualidade deve ser vista como o potencial intrínseco aos atributos do produto ou do serviço de prover satisfação ao cliente. A qualidade percebida, então, depende da função utilidade para o cliente em relação ao nível de desempenho fornecido (ANDERSON; FORNELL, 1994). Por sua vez, a satisfação é um constructo que depende da função valor, podendo ser definida como o quociente entre a qualidade percebida e o preço, ou pela relação entre os benefícios recebidos pelo cliente comparativamente aos custos ou sacrifícios incorridos para sua obtenção (ANDERSON et al., 1992).

Portanto, pode-se entender a qualidade como a percepção atual do cliente sobre o desempenho de um produto ou serviço, e a satisfação como sendo baseada não somente na experiência atual, mas também em experiências passadas, coerentemente com o aspecto cumulativo da definição de satisfação postulado por diversos autores (ANDERSON et al., 1992; JOHNSON; FORNELL, 1991; ANDERSON; FORNELL, 1994; GARBARINO; JOHNSON, 1999), e em experiências futuras, antecipadas pelo julgamento do cliente devido às suas experiências acumuladas, além da projeção das suas próprias expectativas (FORNELL, 1995).

\section{CONCEITO DE IMAGEM}

Muito relacionado aos aspectos intangíveis, o conceito de imagem pode ser analisado a partir de três diferentes perspectivas, porém, inter-relacionadas. Primeiro, as imagens como representações mentais se referem às representações internas ou modelos mentais que são utilizados no processamento de informações (KOSSLYN, 1996). Segundo, as imagens como representações sociais são construções ideológicas, determinadas pela história e pela sociedade (MOSCOVICI, 1978). Terceiro, a imagem na perspectiva do marketing identifica-se como a soma de convicções, atitudes e impressões que uma pessoa ou grupo tem sobre um objeto - serviço ou produto, loja, corporação, marca - (BARICH; KOTLER, 1991; STERN et al., 2001). Cabe salientar que o foco do trabalho está direcionado à imagem como um componente estratégico das ações mercadológicas.

A partir de pesquisa na literatura, Reynolds; Gutman (1984) identificam cinco definições de imagem: (i) um conjunto de características gerais e de sentimentos ou impressões sobre um objeto, sendo que o objeto congrega tanto aspectos da estrutura física, quanto aspectos emocionais; (ii) a percepção do objeto que representa a imagem que se tem em relação a ele; (iii) um conjunto de convicções e atitudes que constituem as imagens fortemente arraigadas na memória; (iv) a personalidade da marca, referindo-se ao conjunto de significados sobre os atribu- 
tos do objeto; e (v) uma combinação de características, sentimentos, emoções e percepções sobre o objeto.

Quando o indivíduo pensa em um serviço, algumas características vêm à sua mente. Essas características formam outras características, construindo uma rede de elementos associados na memória. Com base nessas cinco definições, os mesmos autores também definem imagem como um conjunto de significados hierarquicamente organizados e armazenados na memória, com sua identificação emergindo a partir da rede de relacionamentos entre os diferentes significados ou atributos contidos na memória da pessoa.

Em contrapartida, Poiesz (1989) afirma que é possível distinguir três definições fundamentais de imagem. A primeira abordagem é a de Reynolds; Gutman (1984), que trata a imagem como uma rede de significados armazenados na memória. Esta perspectiva é considerada como de alto nível de elaboração, e a operacionalização das imagens ocorre pela cadeia meio-fim, que significa uma conexão entre atributos do objeto, ações do consumidor e valores pessoais. A segunda abordagem considera a imagem como uma atitude ou combinação de convicções, enquanto que a terceira enfoca as imagens como uma impressão holística ou uma percepção relativa ao objeto entre os competidores.

As diferentes conceituações de imagem mostram que não há uma definição consensual. A falta de convergência quanto às definições de imagens contribui para a multiplicidade de estudos não relacionados. Dessa forma, a definição do constructo de imagem indica que uma abordagem "ambos/e" pode ser mais útil do que uma abordagem "e/ ou", fazendo com que a imagem seja entendida como um todo multidimensional em que suas partes estão intimamente relacionadas. O conceito de imagem congrega idéias, sentimentos, emoções, atitudes, constructos mentais, entendimentos ou expectativas como seus elementos formadores, sendo que a localização da imagem está tanto no mundo externo (influências socioculturais e estímulos) quanto no mundo interno (capacidade cognitiva e emocional do indivíduo). Conseqüentemente, pode-se pressupor que imagem é um conjunto de representações, impressões, convicções e redes de significados de um objeto armazenado na memória de forma holística.

Analisando o atual estágio de pesquisa sobre a Teoria da Imagem, é possível destacar algumas conclusões relevantes (STERN et al., 2001; PARAMESWARAN; PISHARODI, 1994): (i) a imagem desempenha um papel significativo na influência sobre o modo como o público responde ao serviço ou produto, à loja, às organizações e às marcas; (ii) a importância da imagem está arraigada na
Teoria da Imagem, que apregoa que o comportamento humano é direcionado pelas imagens individuais do seu ambiente; (iii) um produto não tem somente uma natureza física, mas também uma natureza social e psicológica e, conseqüentemente, o conjunto de sentimentos, idéias e atitudes é crucial para a tomada de decisão no processo da compra; (iv) imagem é um conceito vital para a gestão mercadológica; (v) a eficiência das variáveis da imagem depende de uma precisa definição e delineamento de seus vários componentes e da conveniência e acuracidade com que estas variáveis são mensuradas; (vi) em função da variedade de definições e técnicas de mensuração, muitos progressos necessitam ser feitos antes que a Teoria da Imagem seja consolidada.

\section{ORGANIZAC̣ÃO DAS IMAGENS E ELEMENTOS CONSTITUINTES}

Toda representação é organizada em torno de um núcleo central, constituído de um ou mais elementos que dão à representação o seu significado, ocupando uma posição privilegiada na estrutura da representação (ABRIC, 1984). O núcleo central constitui-se como um subconjunto da representação, composto de um ou mais elementos cuja ausência desestruturaria a representação ou lhe daria uma significação completamente diferente (SÁ, 1996).

\section{uando o indivíduo pensa em um serviço, características vêm à sua mente, construindo uma rede de elementos associados na memória.}

A organização interna de uma imagem ou representação é regida por um sistema interno duplo em que cada um tem um papel específico, porém, complementar (ABRIC, 1984). Em primeiro lugar, há um sistema central, que é constituído pelo núcleo central da representação, determinado pelas condições históricas, sociais e ideológicas em uma relação dinâmica entre o indivíduo e o objeto. As imagens que compõem o núcleo central são marcadas pela memória coletiva, são estáveis, resistentes à mudança. Sua função é gerar significação para as representações. Por outro lado, o sistema periférico é constituído pelos elementos que estão mais afastados do núcleo central. São elementos mais flexíveis, sensíveis ao contexto imediato. Sua função é permitir a adaptação à realidade concreta, bem como a diferenciação do conteú- 
do e a proteção ao sistema central. Assim, os sistemas periféricos estão mais próximos das práticas do cotidiano e mais suscetíveis às mudanças (SÁ, 1996).

No processo de formação das imagens, além da capacidade perceptiva, entram em jogo o saber, os afetos e as crenças, que são modelados pela vinculação de um espaço cultural e temporal. Nesse contexto, a imagem é constituída por diversas dimensões: tangíveis e intangíveis, mensuráveis e não-mensuráveis, significantes e insignificantes, mutáveis e imutáveis (MAY, 1974). A partir disso, identifica-se que a imagem de serviços é uma construção sistêmica, podendo ser configurada por um conjunto de elementos, conforme mostra a Figura 1.

Os elementos funcionais são traduzidos a partir de uma série de características físicas dos serviços, conferindo-lhes evidências tangíveis tais como instalações de apoio e bens facilitadores, e expressam a utilidade do serviço em si, permitindo aos consumidores atribuir um valor aos serviços prestados (STERN et al., 2001).

Em relação aos elementos simbólicos, os serviços são avaliados pelo que significam ou representam para o indivíduo. O valor simbólico ou a imagem é a extensão que realça o valor da pessoa a seus próprios olhos (auto-estima) e aos olhos dos outros (status). Ao utilizar um serviço, o indivíduo pode não apenas fazer uso das instalações físicas e demais recursos, mas também se identificar com aquilo que a empresa possa lhe oferecer para aumentar sua auto-estima, desde que tal aspecto represente um forte símbolo de status (LEVY, 1959 e 1981; MARTINEAU, 1958).

Os elementos cognitivos são construções mentais sobre o objeto, sendo que o indivíduo avalia a imagem dos serviços como uma impressão e um conjunto de atitudes e percepções sobre o objeto, no caso, a prestação dos serviços em si (MARTINEAU, 1958; DICHTER, 1985), avaliando aspectos relativos ao provedor de serviços (empresa e pessoal de contato direto) e transacionais (relação custo $x$ benefício).

Finalmente, os elementos emocionais, que revelam um conjunto de sentimentos tais como: prazer, dor, alegria e medo, vivenciados pelo indivíduo (REYNOLDS; GUTMAN, 1984; GOLEMAN, 1995; LEDOUX, 2001) à medida que interage nas experiências de produção e consumo dos serviços (característica da simultaneidade dos serviços).

Para facilitar a compreensão dos elementos considerados e ilustrar sua operacionalização, segue o Quadro 1.

É oportuno salientar que as pessoas não apenas registram os objetos e eventos em si, mas também, de forma funcional, simbólica, cognitiva e emocional, ou seja, esses elementos podem ser constituídos concomitantemente na mente dos indivíduos e, desta maneira, a imagem combina um conjunto de fatores tangíveis (funcionais) com outro conjunto de fatores intangíveis (cognitivos, psicológicos e emocionais) (LINDQUIST, 1975).

\section{AMBIÊNCIA DA PESQUISA}

Como campo de pesquisa, foi utilizado o ambiente de serviços de fisioterapia disponibilizado por um Plano de Saúde, classificado como Medicina de Grupo. Essa empresa integra um Grupo de Saúde privado, compreendido por cinco unidades de negócio: o Plano de Saúde, com atuação regional e cerca de 55.000 associados, distribuídos em mais de 1.600 empresas-cliente, demonstrando que o seu foco está no segmento empresarial; Hospital, Medicina do Trabalho, Laboratório e Farmácias.

Como a empresa demonstra preocupação com a satisfação e retenção de seus usuários, justifica-se tal estudo, ainda mais ao abordar os usuários que utilizaram ou estão utilizando os serviços de fisioterapia, uma vez que este

Figura 1: Elementos responsáveis pela configuração de imagens de serviços.

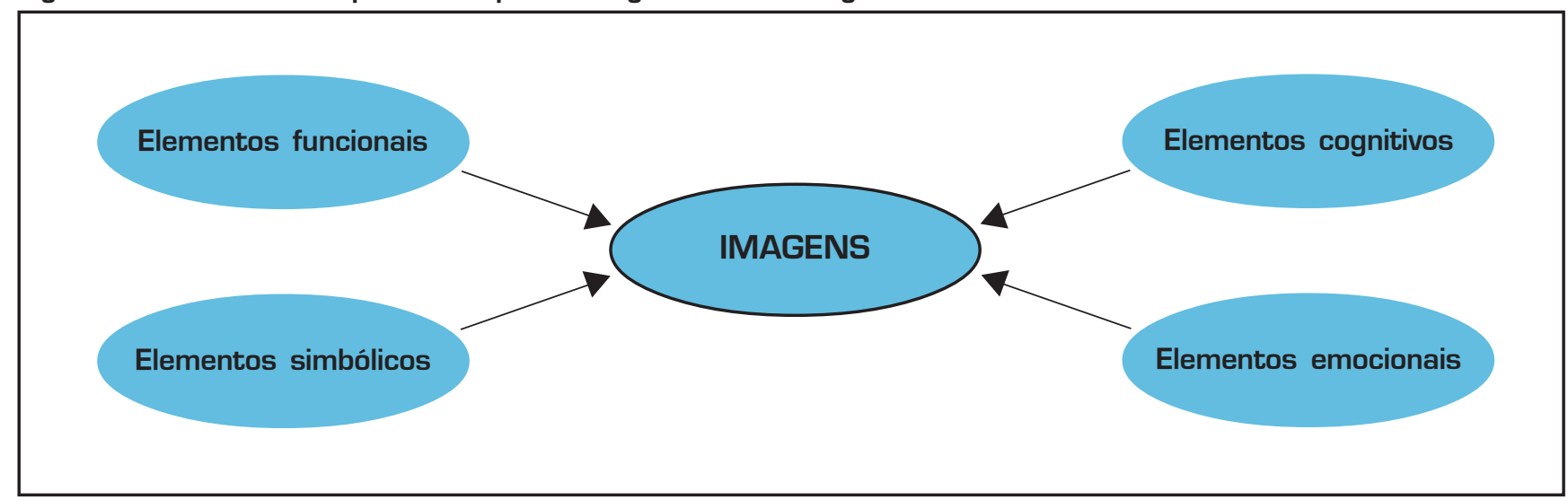

Fonte: Revisão da literatura. 
tipo de serviço apresenta alto grau de interação com os pacientes, os quais denotam altos níveis de envolvimento e expectativa, principalmente relacionados à resolubilidade do tratamento.

Para que os objetivos do estudo pudessem ser alcançados, foram abordados os três tipos de paciente que são encaminhados aos serviços de fisioterapia. A classificação dos pacientes adotada pela empresa é a mesma que a encontrada na literatura (ADLER et al., 1999; STARKEY, 2001; THOMSON et al., 2002):

a. Pós-Operatórios ou Agudos: apresentam alterações agudas em tecidos do corpo que reage desenvolvendo um processo inflamatório na tentativa de remover o elemento irritante. As principais características são: dor, vermelhidão, calor, tumefação e perda da função;

b. Neurológicos: as diferentes partes do sistema nervoso são todas integradas, porém, nem sempre é possível explicar todas as características clínicas a partir dos fatos conhecidos ou da patologia, as quais geralmente são inflamatórias ou degenerativas. As lesões ou doenças do sistema nervoso podem ser originadas por trauma, infecções, condições circulatórias, defeitos congênitos, condições hereditárias, neoplasias ou substâncias tóxicas. As características clínicas das lesões ou doenças podem se manifestar por alteração do estado mental, incapacidade física, movimento involuntário, problemas sensoriais, defeitos visuais, auditivos e de fala e por lesões tróficas;

c. Crônicos: as doenças degenerativas crônicas das articulações constituem-se em uma das causas mais comuns de dor, incapacidade e perdas econômicas ao ser humano. A inflamação crônica pode sobrevir a uma reação aguda quando a defesa do organismo é insuficiente para permitir a resolução de um problema e há uma infecção persistente de alto grau ou um processo irritativo. A dor, quando crônica, é resistente ao tratamento padronizado, o que exige do profissional o conhecimento de outros aspectos sobre o paciente, aspectos físicos, psíquicos e sua relação com a família e a sociedade.

A fim de contextualizar sobre os serviços de fisioterapia em estudo e o público-alvo da pesquisa, destacam-se alguns dados, conforme mostra a Tabela 1. Considerando o ano de 2003, em média, foram 3.094 atendimentos/ mês, totalizando 37.123 atendimentos/ano, dentre os três tipos de paciente. Observa-se que os pacientes pós-operatórios representam $44,40 \%$ sobre o total de atendimentos (número de sessões), enquanto que os neurológicos $9,25 \%$ e os crônicos $46,35 \%$. Ao todo, foram atendidos cerca de 2.300 pacientes.

\section{MÉTODO DA PESQUISA}

Para a pesquisa, foram estabelecidos os seguintes objetivos: a) identificar as imagens dos serviços de fisioterapia na percepção de seus usuários, considerando os três tipos de paciente identificados, bem como analisar a composição das imagens no que se refere ao seu conjunto de elementos; b) verificar a organização interna dos atributos relacionados à imagem dos serviços; e c) avaliar alguns aspectos atinentes ao desempenho do provedor de serviços.

$\mathrm{O}$ método de pesquisa adotado compreende procedimentos desenvolvidos para acessar a configuração das

\section{Quadro 1: Exemplos dos elementos que configuram as imagens de serviços.}

\begin{tabular}{|c|c|}
\hline ELEMENTOS & EXEMPLOS \\
\hline Funcionais & Aspectos tangíveis dos serviços de fisioterapia (instalações, equipamentos...J. \\
\hline Simbólicos & $\begin{array}{l}\text { Melhoria na auto-estima do paciente ao voltar às atividades normais (trabalho, prática de } \\
\text { esportes,...). }\end{array}$ \\
\hline Cognitivos & $\begin{array}{l}\text { Relação entre o resultado (alívio da dor, recuperação...) do tratamento de fisioterapia e a } \\
\text { mensalidade paga ao Plano de Saúde (relação custo } x \text { benefícios). }\end{array}$ \\
\hline Emocionais & $\begin{array}{l}\text { Sentimento de alegria à medida que o paciente alivia suas dores e diminui suas limitações } \\
\text { motoras. }\end{array}$ \\
\hline
\end{tabular}

Tabela 1: Atendimentos por tipo de paciente.

\begin{tabular}{|l|c|c|c|}
\hline \multicolumn{1}{|c|}{ TIPO DE PAGIENTE } & ATENDIMENTOS/ANO & ATENDIMENTOS/MÊS & REPRESENTATIVIDADE (\%) \\
\hline Pós-Operatórios ou Agudos & 16.483 & 1.374 & 44,40 \\
\hline Neurológicos & 3.432 & 286 & 9,25 \\
\hline Crônicos & 17.208 & 1.434 & 46,35 \\
\hline TOTAL & $\mathbf{3 7 . 1 2 3}$ & $\mathbf{3 . 0 9 4}$ & $\mathbf{1 0 0}$ \\
\hline
\end{tabular}

Fonte: Relatório de Atendimentos da Fisioterapia (2003). 
representações ou imagens (ABRIC, 1984 e 1993; MOSCOVICI, 1978; SÁ, 1996 e 1998; SCHULER, 2000; DE TONI; SCHULER, 2003). A partir de procedimentos propostos pela literatura, foi implementado um método para a configuração de imagens de serviços, com fins exploratórios.

do tratamento, se caracterizam, principalmente, pela freqüência de uso Baixa e Moderada. Observou-se que os pacientes neurológicos, até pelo fato de apresentarem sintomas permanentes e/ou de evolução em suas patologias, incidem em uma freqüência de uso Alta. Os pacientes crônicos, por retornarem, sistematicamente, ao tratamento, caracterizam-se pela freqüência de uso Moderada, embora as freqüências Baixa e Alta também se mostrem significativas. Destaca-se que o critério de seleção da amostra foi por conveniência, embora tenham sido mantidos os critérios de representatividade do perfil dos usuários dos serviços.

A coleta de dados foi realizada por meio de entrevistas individuais, utilizan-

Tal método contempla duas abordagens: uma qualitativa, voltada à identificação dos atributos, a qual foi implementada por meio do tratamento dos dados coletados nas entrevistas (Análise de Conteúdo); e outra quantitativa, direcionada à organização dos atributos, e seus respectivos elementos, em torno do núcleo central das imagens (EVOC), além da aplicação de uma Escala de Diferencial Semântico e uma Escala de Satisfação, ampliando o entendimento a respeito da percepção dos pacientes quanto aos serviços prestados.

O público-alvo para a composição amostral foi composto por pacientes que utilizaram os serviços de fisioterapia prestados pelo Plano de Saúde no período de fevereiro a abril de 2004. Foram entrevistados 38 pacientes, caracterizados conforme a Tabela 2. Para que a amostra fosse representativa em relação ao perfil dos usuários dos serviços, identificou-se, em conjunto com a Coordenadora do Setor, além da análise de relatórios, os aspectos que deveriam ser observados (sexo dos respondentes e freqüência de uso dos serviços).

Quanto à freqüência de uso, o Plano de Saúde adota os seguintes critérios em relação ao número de sessões/ano: (1) Baixa (até 20); (2) Moderada (de 20 a 60); e (3) Alta (mais de 60). Para a composição da amostra em relação à freqüência de uso, alguns cuidados foram necessários, uma vez que os pacientes pós-operatórios, pela natureza do-se um roteiro de questões (Quadro 2). As questões que compõem o roteiro foram desenvolvidas a partir da revisão da literatura e previamente testadas. No pré-teste realizado para a validação do roteiro de questões, o instrumento de coleta de dados foi aplicado, aleatoriamente, a 5 respondentes junto ao ambiente pesquisado, no intuito de verificar o entendimento das questões, sendo que nenhuma alteração foi necessária.

Para qualificar o levantamento dos dados, as entrevistas foram conduzidas por uma psicóloga devidamente treinada, utilizando a técnica da livre associação. Essa técnica consiste em apresentar aos entrevistados um estímulo ou termo indutor (fisioterapia), solicitando que digam quais palavras ou expressões lhes vêm imediatamente à lembrança. A principal vantagem da técnica é que permite a identificação dos elementos latentes na mente das pessoas sobre o termo indutor sem exercer um controle exagerado da racionalidade. Sendo assim, foi possível identificar os elementos funcionais, simbólicos, cognitivos e emocionais em relação ao objeto investigado (POIESZ, 1989; SÁ, 1996; MALHOTRA, 2001).

Para avaliar, de forma complementar, alguns atributos relacionados ao desempenho do provedor de serviços, foi proposta uma Escala de Diferencial Semântico (Quadro 3), desenvolvida a partir da revisão da literatura, contando, também, com a participação de um profissional liga-

Tabela 2: Perfil dos respondentes.

\begin{tabular}{|c|c|c|c|c|c|c|}
\hline \multirow{2}{*}{$\begin{array}{l}\text { TIPO DE } \\
\text { PACIENTE }\end{array}$} & \multirow{2}{*}{$\begin{array}{l}\text { ENTREVISTADOS POR } \\
\text { TIPO DE PAGIENTE }\end{array}$} & \multicolumn{2}{|c|}{ SEXO } & \multicolumn{3}{|c|}{ FREQÜÊNCIA DE USO } \\
\hline & & MASCULINO & FEMININO & 1 & 2 & 3 \\
\hline Pós-Operatórios ou Agudos & 14 & 6 & 8 & 7 & 5 & 2 \\
\hline Neurológicos & 10 & 5 & 5 & - & - & 10 \\
\hline Crônicos & 14 & 6 & 8 & 3 & 8 & 3 \\
\hline TOTAL & 38 & - & - & & & \\
\hline
\end{tabular}

Fonte: Relatório de Atendimentos da Fisioterapia (2003). 
do ao Plano de Saúde e da Coordenadora do Setor de Fisioterapia. Além da referida escala, foi acrescentada uma questão relativa à satisfação geral do usuário em relação aos serviços de fisioterapia, a qual apresenta uma escala do tipo Likert de sete pontos.

Após a entrevista individual, mediante o roteiro de questões, foi entregue, para cada entrevistado, um instrumento de coleta de dados composto pela Escala de Diferencial Semântico e pela questão de satisfação geral. Tal instrumento foi preenchido individualmente e devolvido ao entrevistador. A duração média das entrevistas individuais e do preenchimento da Escala e da questão sobre a satisfação geral foi de 8 minutos para os pacientes pósoperatórios e crônicos. Os pacientes neurológicos, por apresentarem uma maior dificuldade devido às suas limitações, utilizaram, em média, 12 minutos.
O tratamento dos dados foi realizado em três etapas. A primeira etapa foi constituída da análise de conteúdo das respostas geradas a partir do roteiro de questões (Quadro 2). A "análise de conteúdo consiste num conjunto de técnicas de análise das comunicações visando obter, por procedimentos sistemáticos e objetivos de descrição do conteúdo das mensagens, indicadores (quantitativos ou não) que permitam a inferência de conhecimentos relativos às condições de produção/recepção (variáveis inferidas) destas mensagens" (BARDIN, 2002, p. 42). Salienta-se que uma boa análise de conteúdo parte de um bom problema de pesquisa e de categorias de análise previamente definidas (QUEZADA, 1992). Inicialmente, foram listadas as idéias mencionadas pelos respondentes, gerando os atributos pertinentes à imagem relacionada aos serviços de fisioterapia para cada tipo de paciente. Em

\section{Quadro 2: Roteiro de questões.}

ROTEIRO DE QUESTÕES

1. Quando eu digo FISIOTERAPIA, qual a primeira coisa que lhe vem à cabeça?

2. Que outras idéias lhe vêm à mente sobre FISIOTERAPIA?

3. Como você descreve a FISIOTERAPIA?

4. Qual o significado, o que representa a FISIOTERAPIA para você?

5. Qual o sentimento que lhe vem à mente quando eu digo FISIOTERAPIA?

6. Qual o benefício que a FISIOTERAPIA traz para você?

Quadro 3: Escala de Diferencial Semântico e questão sobre satisfação geral. ESCALA DE DIFERENCIAL SEMÂNTICO

Na sua opinião, como você avalia os seguintes atributos (características) em relação ao Serviço de Fisioterapia prestado pelo Plano de Saúde? Assinale com um X em cima de uma das sete posições (__ ) alternativas:

\section{Desorganizado \\ Não pontual \\ Desatencioso \\ Mal equipado \\ Ineficiente}

Não auxilia na recuperação

Não alivia a dor

Frio

Inseguro

Não confiável

Não recomendável
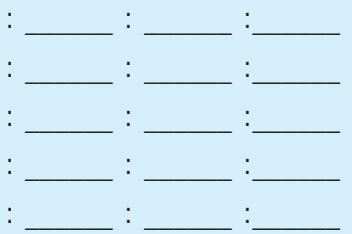

:

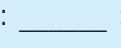

:___ Organizado

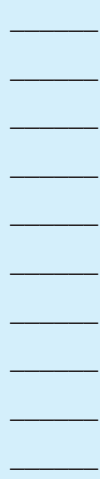

Pontual

Atencioso

Bem equipado

Eficiente

Auxilia na recuperação

Alivia a dor

Caloroso

Seguro

Confiável

Recomendável

Qual é o seu grau de satisfação geral com o Serviço de Fisioterapia do Plano de Saúde?

1.

Totalmente

Insatisfeito
2.
3.
Nem Insatisfeito Nem Satisfeito

5.

6.

7.

Totalmente

Satisfeito 
seguida, os atributos mencionados foram categorizados com base nos quatro elementos da imagem dos serviços.

$\mathrm{Na}$ segunda etapa, os atributos gerados foram analisados quanto à sua frequiência e ordem de evocação. Com esses dois critérios, os atributos foram analisados utilizando-se um software adequado, desenvolvido por Vergès (1992) e seus colaboradores, o EVOC. Cabe ressaltar que o EVOC permite a análise de evocações, buscando, a partir da combinação da frequiência de evocação e ordem média de evocação de cada palavra, identificar as palavras que apresentam maior probabilidade de pertencerem ao núcleo central e ao sistema periférico das imagens.
$(61,83 \%)$, representado, principalmente, pelos atributos "recuperação" $(31,30 \%)$ e "alívio da dor" $(11,45 \%)$; seguido dos elementos funcionais $(20,61 \%)$, emocionais $(10,69 \%)$ e simbólicos $(6,87 \%)$. No caso do elemento funcional, o atributo de maior destaque é "exercícios" $(14,50 \%)$. Para o elemento emocional, emergiu o atributo "bem-estar" $(6,11 \%)$ e, para o simbólico, o atributo "dedicação (empenho)" (3,05\%).

Em relação aos pacientes neurológicos, o elemento mais significativo para a construção da imagem é o cognitivo $(40,00 \%)$, representado, principalmente, pelo atributo "recuperação" (30,00\%); seguido dos elementos emocional $(28,00 \%)$, simbólico $(18,00 \%)$ e funcional $(14,00 \%)$. No caso do elemento emocional, os atributos de maior destaque são "atenção e carinho" (11,00\%) e "bem-estar" $(9,00 \%)$. Para o elemento simbólico, os atributos mais evocados são "volta à vida normal" $(6,00 \%)$ e "dedicação (empenho)" $(4,00 \%)$ e, para o funcional, o principal atributo é "exercícios" $(9,00 \%)$.

Finalmente, na terceira etapa, foi realizada a análise estatística das respostas dos entrevistados provenientes da Escala de Diferencial Semântico e da questão relativa ao grau de satisfação geral.

\section{IDENTIFICAC̄̃̃O DOS ATRIBUTOS E SEUS ELEMENTOS CONSTITUINTES}

Mediante análise de conteúdo dos dados coletados nas entrevistas individuais, foram identificados os atributos relacionados à imagem dos serviços de fisioterapia. De acordo com os direcionamentos encontrados na literatura, a imagem é uma construção sistêmica, podendo ser configurada por meio de uma gama de elementos funcionais, simbólicos, cognitivos e emocionais. Dessa forma, os atributos relacionados à construção da imagem dos serviços de fisioterapia, na perspectiva de seus usuários, e seus respectivos elementos, são apresentados no Quadro 4, onde VF representa o Valor de Freqüência.

Inicialmente, percebe-se que há diferença entre os tipos de paciente no que diz respeito aos elementos e atributos responsáveis pela construção da imagem dos serviços de fisioterapia. De forma geral, nota-se predominância dos elementos cognitivos $(48,80 \%)$ com seus respectivos atributos, em relação aos demais elementos, ou seja, elementos emocionais $(20,80 \%)$, funcionais $(20,53 \%)$ e simbóli$\cos (9,87 \%)$. Consoante a isso, verifica-se que tais diferenças merecem uma análise mais aprofundada.

Para os pacientes pós-operatórios, o elemento mais significativo para a construção da imagem é o cognitivo
Para os pacientes crônicos, o elemento mais significativo para a construção da imagem é o cognitivo $(43,06 \%)$, caracterizado, em essência, pelos atributos "alívio da dor" $(15,28 \%)$ e "recuperação" (13,89\%); seguido dos elementos funcional e emocional (25,00\% cada), e simbólico $(6,94 \%)$. No caso do elemento funcional, os principais atributos identificados foram "exercícios" $(13,19 \%)$ e "aparelhos" (6,25\%). Quanto ao elemento emocional, destaca-se o atributo "bem-estar" $(13,19 \%)$ e, para o simbólico, o atributo "dedicação (empenho)" (3,47\%).

\section{ORGANIZACÃO DOS ATRIBUTOS EM RELAC̣ÃOO AO TERMO INDUTOR}

Com a identificação dos atributos relacionados à construção da imagem dos serviços de fisioterapia, além da frequiência, foi analisada a ordem de evocação. Utilizando-se tais critérios, os dados foram processados com o suporte do software EVOC, obtendo-se as imagens centrais, intermediárias e periféricas, de acordo com o Valor de Freqüência (VF) e o Valor de Ordem (VO). Os VF's e VO's são dispostos em um gráfico com quatro quadrantes. O primeiro quadrante corresponde ao Núcleo ou Imagem Central; o segundo, à primeira imagem intermediária; o terceiro, à segunda imagem intermediária; e o quarto, à imagem periférica.

Os critérios de classificação são os seguintes (VERGÈS, 1992): a) Núcleo ou Imagem Central: atributos com VO menor que a média das médias das evocações, denominado Valor de Ordem Médio (VOM), e VF 
maior ou igual ao Valor de Freqüência Médio (VFM) das evocações; b) Primeira Imagem Intermediária: atributos com VO maior ou igual ao VOM, e VF maior ou igual ao VFM das evocações; c) Segunda Imagem Intermediária: atributos com VO menor que o VOM, e VF menor ao VFM das evocações; e d) Imagem Periférica: atributos com VO maior ou igual ao VOM, e VF menor que o VFM das evocações.

O VFM é calculado pelo total de freqüência de atributos citados, dividido pelo número de diferentes atributos $($ Ex. VFM Geral $=375 / 25=15)$. O VO e o VOM são calculados a partir da freqüência dos atributos na memó- ria dos entrevistados. $\mathrm{O}$ atributo citado em primeiro lugar recebe peso 1 , quando for citado em segundo lugar, peso 2, e assim sucessivamente até o último atributo citado. Finalmente, com um somatório simples chega-se ao VO. Por exemplo, o atributo "recuperação" foi citado 91 vezes, sendo assim distribuído: $(16 \times 1)+(6 \times 2)+(8 \times 3)+$ $(6 \times 4)+(9 \times 5)+(14 \times 6)+(10 \times 7)+(5 \times 8)+(6 \times 9)+(4 \times 10)$ $+(2 \times 11)+(2 \times 12)+(1 \times 13)+(2 \times 15)$, obtendo-se o Valor de Evocação igual a 498, que dividido pela frequiência (91), resulta no VO $=5,473$. Já o VOM é calculado a partir da soma dos Valores de Evocações dividida pelo total de frequiência de citações (Ex: VOM Geral

Quadro 4: Identificação dos atributos e seus elementos constituintes.

\begin{tabular}{|c|c|c|c|c|c|c|c|c|c|}
\hline \multirow{2}{*}{ ELEMENTOS } & \multirow[b]{2}{*}{ ATRIBUTOS } & \multicolumn{2}{|c|}{ GERAL } & \multicolumn{2}{|c|}{ PÓS-OPERATÓRIOS } & \multicolumn{2}{|c|}{ NEUROLÓGICOS } & \multicolumn{2}{|c|}{ CRÔNICOS } \\
\hline & & VF & $\%$ & VF & $\%$ & VF & $\%$ & VF & $\%$ \\
\hline \multirow[t]{6}{*}{ Funcionais } & Exercícios & 47 & 12,53 & 19 & 14,50 & 9 & 9,00 & 19 & 13,19 \\
\hline & Aparelhos & 11 & 2,93 & 1 & 0,76 & 1 & 1,00 & 9 & 6,25 \\
\hline & Orientações & 10 & 2,67 & 5 & 3,82 & 1 & 1,00 & 4 & 2,78 \\
\hline & Tratamento & 7 & 1,87 & 2 & 1,53 & 2 & 2,00 & 3 & 2,08 \\
\hline & Sistemática & 2 & 0,53 & 0 & 0,00 & 1 & 1,00 & 1 & 0,69 \\
\hline & Subtotal & 77 & 20,53 & 27 & 20,61 & 14 & 14,00 & 36 & 25,00 \\
\hline \multirow[t]{7}{*}{ Simbólicos } & Dedicação (empenho) & 13 & 3,47 & 4 & 3,05 & 4 & 4,00 & 5 & 3,47 \\
\hline & Volta à vida normal & 7 & 1,87 & 1 & 0,76 & 6 & 6,00 & 0 & 0,00 \\
\hline & Satisfação (resultado) & 6 & 1,60 & 2 & 1,53 & 2 & 2,00 & 2 & 1,39 \\
\hline & Volta ao trabalho & 5 & 1,33 & 1 & 0,76 & 3 & 3,00 & 1 & 0,69 \\
\hline & Qualidade de vida & 3 & 0,80 & 0 & 0,00 & 1 & 1,00 & 2 & 1,39 \\
\hline & Auto-estima & 3 & 0,80 & 1 & 0,76 & 2 & 2,00 & 0 & 0,00 \\
\hline & Subtotal & 37 & $\mathbf{9 , 8 7}$ & 9 & 6,87 & 18 & 18,00 & 10 & 6,94 \\
\hline \multirow[t]{9}{*}{ Cognitivos } & Recuperação & 91 & 24,27 & 41 & 31,30 & 30 & 30,00 & 20 & 13,89 \\
\hline & Alívio da dor & 39 & 10,40 & 15 & 11,45 & 2 & 2,00 & 22 & 15,28 \\
\hline & Possibilidade de cura & 15 & 4,00 & 5 & 3,82 & 4 & 4,00 & 6 & 4,17 \\
\hline & Auxílio (recursos) & 15 & 4,00 & 10 & 7,63 & 1 & 1,00 & 4 & 2,78 \\
\hline & Rapidez na recuperação & 8 & 2,13 & 3 & 2,29 & 0 & 0,00 & 5 & 3,47 \\
\hline & Solução de um problema & 6 & 1,60 & 4 & 3,05 & 0 & 0,00 & 2 & 1,39 \\
\hline & Algo necessário & 5 & 1,33 & 3 & 2,29 & 0 & 0,00 & 2 & 1,39 \\
\hline & Melhora na saúde & 4 & 1,07 & 0 & 0,00 & 3 & 3,00 & 1 & 0,69 \\
\hline & Subtotal & 183 & 48,80 & 81 & 61,83 & 40 & 40,00 & 62 & 43,06 \\
\hline \multirow[t]{8}{*}{ Emocionais } & Bem-estar & 36 & 9,60 & 8 & 6,11 & 9 & 9,00 & 19 & 13,19 \\
\hline & Atenção e carinho & 16 & 4,27 & 0 & 0,00 & 11 & 11,00 & 5 & 3,47 \\
\hline & Amizade & 10 & 2,67 & 0 & 0,00 & 5 & 5,00 & 5 & 3,47 \\
\hline & Dor & 8 & 2,13 & 3 & 2,29 & 0 & 0,00 & 5 & 3,47 \\
\hline & Mal-Estar & 5 & 1,33 & 3 & 2,29 & 1 & 1,00 & 1 & 0,69 \\
\hline & Limitação & 3 & 0,80 & 0 & 0,00 & 2 & 2,00 & 1 & 0,69 \\
\hline & Subtotal & 78 & 20,80 & 14 & 10,69 & 28 & 28,00 & 36 & 25,00 \\
\hline & TOTAL & 375 & 100,00 & 131 & 100,00 & 100 & 100,00 & 144 & 100,00 \\
\hline
\end{tabular}


= 2201/375 = 5,9) (Quadro 5). Dessa forma, o VFM corresponde a 15 no geral, 7 para os pós-operatórios, 5 para os neurológicos e 6 para os crônicos. O VOM corresponde a 5,9 no geral, 5,3 para os pós-operatórios, 6,3 para os neurológicos e 6,2 para os crônicos.

Em relação ao Núcleo ou Imagem Central dos serviços de fisioterapia, analisando-se globalmente, sem haver a segmentação por tipo de paciente, foram identificados os atributos "recuperação" (VF = 91 e VO = 5,473), "exercícios" (VF $=47$ e VO = 4,064) e "auxílio (recursos)" $(\mathrm{VF}=15$ e $\mathrm{VO}=5,000)$.

Para os pacientes pós-operatórios, o Núcleo ou Imagem Central está relacionado aos atributos "recuperação" (VF
$=41$ e VO $=5,220)$ e "exercícios" $(\mathrm{VF}=19$ e $\mathrm{VO}=4,000)$. A Primeira Imagem Intermediária está representada pelos atributos "alívio da dor" (VF $=15$ e VMO $=5,800)$, "auxílio (recursos)" $(\mathrm{VF}=10$ e $\mathrm{VO}=5,700)$ e "bem-estar" $(\mathrm{VF}=8$ e VO $=6,125)$; e a Segunda Imagem Intermediária, principalmente, ao atributo "dedicação (empenho)" $(\mathrm{VF}=4 \mathrm{e}$ VO 5,250). A Imagem Periférica foi representada, em essência, pelos atributos "possibilidade de cura" $(\mathrm{VF}=5$ e $\mathrm{VO}=6,400)$, "orientações" ( $\mathrm{VF}=5$ e $\mathrm{VO}=$ $5,400)$ e "solução de um problema" ( $\mathrm{VF}=4$ e $\mathrm{VO}=6,500)$.

Para os pacientes neurológicos, o Núcleo ou Imagem Central está relacionado aos atributos "recuperação" (VF $=30$ e $\mathrm{VO}=5,400)$ e "exercícios" $(\mathrm{VF}=9$ e VO $=5,111)$.

Quadro 5: Organização das imagens dos serviços de fisioterapia.

\begin{tabular}{|c|c|c|c|c|c|c|c|c|c|c|c|}
\hline \multicolumn{12}{|c|}{ IMAGEM CENTRAL } \\
\hline \multicolumn{3}{|c|}{ GERAL } & \multicolumn{3}{|c|}{ PÓS-OPERATÓRIOS } & \multicolumn{3}{|c|}{ NEUROLÓGICOS } & \multicolumn{3}{|c|}{ CRÔNICOS } \\
\hline \multicolumn{3}{|c|}{$V F>=15$ e $V O<5,9$} & \multicolumn{3}{|c|}{$V F>=7$ e $V O<5,3$} & \multicolumn{3}{|c|}{$V F>=5$ e $V O<6,3$} & \multicolumn{3}{|c|}{$\mathrm{VF}>=6$ e $\mathrm{VO}<6,2$} \\
\hline Atributos & VF & Vo & Atributos & VF & VO & Atributos & VF & VO & Atributos & VF & Vo \\
\hline Auxílio & 15 & 5,000 & Exercícios & 19 & 4,000 & Exercícios & 9 & 5,111 & Aparelhos & 9 & 4,222 \\
\hline Exercícios & 47 & 4,064 & Recuperação & 41 & 5,220 & Recuperação & 30 & 5,400 & Exercícios & 19 & 3,632 \\
\hline Recuperação & 91 & 5,473 & & & & & & & Recuperação & 20 & 6,100 \\
\hline \multicolumn{12}{|c|}{ PRIMEIRA IMAGEM INTERMEDIÁRIA } \\
\hline \multicolumn{3}{|c|}{ GERAL } & \multicolumn{3}{|c|}{ PóS-OPERATÓRIOS } & \multicolumn{3}{|c|}{ NEUROLÓGICOS } & \multicolumn{3}{|c|}{ CRÔNICOS } \\
\hline \multicolumn{3}{|c|}{$\mathrm{VF}>=15$ e $\mathrm{VO}>=5,9$} & \multicolumn{3}{|c|}{$\mathrm{VF}>=7$ e $\mathrm{VO}>=5,3$} & \multicolumn{3}{|c|}{$\mathrm{VF}>=5$ e $\mathrm{VO}>=6,3$} & \multicolumn{3}{|c|}{$\mathrm{VF}>=6$ e $\mathrm{VO}>6,2$} \\
\hline Atributos & VF & Vo & Atributos & VF & Vo & Atributos & VF & Vo & Atributos & VF & Vo \\
\hline Alívio da dor & 39 & 7,205 & Alívio da dor & 15 & 5,800 & Amizade & 5 & 9,600 & Alívio da dor & 22 & 8,182 \\
\hline Atenção-Carinho & 16 & 7,750 & Auxílio & 10 & 5,700 & Atenção-Carinho & 11 & 7,000 & Bem-estar & 19 & 6,368 \\
\hline Bem-estar & 36 & 6,528 & Bem-estar & 8 & 6,125 & Bem-estar & 9 & 7,222 & Possib. cura & 6 & 7,833 \\
\hline Possib. cura & 15 & 6,867 & & & & Volta à vida & 6 & 8,333 & & & \\
\hline \multicolumn{12}{|c|}{ SEGUNDA IMAGEM INTERMEDIARIA } \\
\hline \multicolumn{3}{|c|}{ GERAL } & \multicolumn{3}{|c|}{ PÓS-OPERATÓRIOS } & \multicolumn{3}{|c|}{ NEUROLÓgICOS } & \multicolumn{3}{|c|}{ CRÔNICOS } \\
\hline \multicolumn{3}{|c|}{$V F<15$ e $V O<5,9$} & \multicolumn{3}{|c|}{$V F<7$ e $V O<5,3$} & \multicolumn{3}{|c|}{$V F<5$ e $V O<6,3$} & \multicolumn{3}{|c|}{$\mathrm{VF}<6$ e $\mathrm{VO}<6,2$} \\
\hline Atributos & VF & Vo & Atributos & VF & Vo & Atributos & VF & Vo & Atributos & VF & Vo \\
\hline Aparelhos & 11 & 4,364 & Dedicação & 4 & 5,250 & Possib. Cura & 4 & 6,000 & Dor & 5 & 4,000 \\
\hline Orientações & 10 & 5,300 & Dor & 3 & 4,000 & Dedicação & 4 & 5,500 & Orientações & 4 & 5,500 \\
\hline Dor & 8 & 4,000 & Rapidez na rec. & 3 & 4,000 & Volta ao trab. & 3 & 2,000 & Auxílio & 4 & 3,750 \\
\hline Tratamento & 7 & 3,429 & Necessário & 3 & 4,333 & Tratamento & 2 & 2,000 & Tratamento & 3 & 4,667 \\
\hline \multicolumn{12}{|c|}{ IMAGEM PERIFÉRIGA } \\
\hline GER & & & PÓS-OPERt & ATÓRI & I0s & NEUROLó & JGICO & & GRố & & \\
\hline$V F<15$ e $V$ & $0>=$ & 5,9 & $\mathrm{VF}<7$ e $\mathrm{VO}$ & $>=5$ & 5,3 & $V F<5$ e $V C$ & D > = & 6,3 & $V F<6$ e $V c$ & $>=$ & 6,2 \\
\hline Atributos & VF & VO & Atributos & VF & VO & Atributos & VF & VO & Atributos & VF & Vo \\
\hline Dedicação & 13 & 6,154 & Possib. cura & 5 & 6,400 & Satisfação & 2 & 9,000 & Amizade & 5 & 8,600 \\
\hline Amizade & 10 & 9,100 & Orientações & 5 & 5,400 & Alívio da dor & 2 & 7,000 & Dedicação & 5 & 7,400 \\
\hline Volta à vida & 7 & 8,286 & Sol. de um problema & 4 & 6,500 & Melhoria da saúde & 3 & 6,333 & Atenção-Carinho & 5 & 9,400 \\
\hline Rapidez na rec. & 8 & 6,250 & \begin{tabular}{|l|} 
Mal-estar \\
\end{tabular} & 3 & 6,67 & Auto-estima & 2 & 12,000 & Rapidez na recup. & 5 & 7,600 \\
\hline
\end{tabular}


A Primeira Imagem Intermediária está representada pelos atributos "atenção e carinho" ( VF = 11 e VO = 7,000), "bem-estar" ( VF = 9 e VO = 7,222), "volta à vida normal" $(\mathrm{VF}=6$ e $\mathrm{VO}=8,333)$ e "amizade" ( VF = 5 e $\mathrm{VO}=9,600)$; e a Segunda Imagem Intermediária, principalmente, aos atributos "possibilidade de cura" (VF $=4$ e VO $=6,000)$ e "dedicação (empenho)" (VF= 4 e VO= 5,500). A Imagem Periférica foi representada, em essência, pelo atributo "melhoria da saúde" (VF $=3$ e VO = 6,333).

Finalmente, para os pacientes crônicos, o Núcleo ou Imagem Central está relacionado aos atributos "recuperação" (VF = 20 e VO = 6,100), "exercícios" (VF = 19 e VO $=3,632)$ e "aparelhos" ( $\mathrm{VF}=9$ e $\mathrm{VO}=4,222)$. A Primeira Imagem Intermediária está representada pelos atributos "alívio da dor" (VF = 22 e VO= 8,182), "bem-estar" (VF $=19$ e $\mathrm{VO}=6,368)$ e "possibilidade de cura" $(\mathrm{VF}=6 \mathrm{e} \mathrm{VO}$ =7,833); e a Segunda Imagem Intermediária, principalmente, aos atributos “dor" (VF $=5$ e VO = 4,000), "auxílio (recursos)" ( VF $=4$ e $\mathrm{VO}=3,750)$ e "orientações" (VF = 4 e $\mathrm{VO}=5,500)$. A Imagem Periférica foi representada, em essência, pelos atributos "amizade" ( VF $=5$ e $\mathrm{VO}=$ 8,600), "atenção e carinho" ( VF = 5 e VO = 9,400), "dedicação (empenho)" ( VF = 5 e VO =7,400) e "rapidez na recuperação" $(\mathrm{VF}=5$ e $\mathrm{VO}=7,600)$.

\section{QUALIDADE PERCEBIDA PELOS USUÁRIOS DOS SERVIC̣OS}

Com o objetivo de avaliar alguns atributos relacionados ao desempenho do provedor de serviços, utilizou-se a Escala de Diferencial Semântico. A utilização desta escala é significativa em relação ao estudo de imagens, pois ajuda a identificar como o serviço ou o provedor de serviços é percebido pelos usuários, fornecendo subsídios importantes para o processo de tomada de decisões.
Conforme a Tabela 3 , os resultados indicam que o Setor de Fisioterapia do Plano de Saúde, na visão de seus usuários, é avaliado como: organizado, pontual, atencioso, bem equipado, eficiente, como algo que auxilia na recuperação, que alivia a dor, caloroso, seguro, confiável e recomendável.

Com base nos resultados provenientes da Escala de Diferencial Semântico, com o objetivo de reduzir a dimensionalidade dos atributos avaliados, procedeu-se a uma Análise Fatorial, utilizando-se a rotação de dados Varimax. No processo de extração dos fatores, algumas medidas indicaram a validade e adequação de tal procedimento, como é o caso da Medida Kaiser-Meyer-Olkin (KMO) de Adequação da Amostra = 0,719; Teste de Esfericidade de Bartlett $=270,057$; Significância $=$ 0,000; e Variância explicada pelos três fatores $=75,23 \%$. Para verificar a confiabilidade dos dados, procedeu-se, também, à aferição do Alfa de Cronbach, o qual resultou para os três fatores, respectivamente, em: 0,9345, 0,7524 e 0,3900. O Fator 3 não obteve um indicador adequado, sendo que tal coeficiente pode ter sido influenciado pelo baixo número de itens na escala (MALHOTRA, 2001).

Sendo assim, foram identificados os seguintes fatores:

F1 - Confiabilidade: segurança, atenção, recomendação, confiança, pontualidade e organização;

F2 - Resolubilidade: auxílio, eficiência e alívio da dor; e F3 - Recursos Técnicos e Humanos: equipamentos e atendimento caloroso.

Por outro lado, os atributos positivos relacionados ao Setor de Fisioterapia também se refletem no grau de satisfação geral dos pacientes (Tabela 4), ou seja, os usuários dos serviços de fisioterapia declinaram um nível de satisfação entre "muito satisfeito" e "totalmente satisfeito" com os serviços prestados.

Tabela 3: Desempenho do Setor de Fisioterapia.

\begin{tabular}{|l|l|l|}
\hline \multicolumn{1}{|c|}{ ATRIBUTOS } & MÉEIA & DESVIO-PADR ÃO \\
\hline 1.Desorganizado / 7.Organizado & 6,48 & 0,87 \\
\hline 1.Não pontual / 7. Pontual & 6,48 & 0,87 \\
\hline 1.Desatencioso / 7.Atencioso & 6,66 & 0,69 \\
\hline 1.Mal equipado / 7. Bem equipado & 5,93 & 1,14 \\
\hline 1.Ineficiente / 7.Eficiente & 6,39 & 0,96 \\
\hline 1.Não auxilia na recuperação / 7.Auxilia na recuperação & 6,36 & 0,99 \\
\hline 1.Não alivia a dor / 7.Alivia a dor & 6,48 & 0,87 \\
\hline 1.Frio / 7.Caloroso & 6,81 & 0,63 \\
\hline 1.Inseguro / 7.Seguro & 6,69 & 0,63 \\
\hline 1.Não confiável / 7.Confiável & 6,78 & 0,65 \\
\hline 1.Não recomendável / 7.Recomendável & 6,66 & 0,77 \\
\hline
\end{tabular}


Tabela 4: Grau de satisfação geral.

\begin{tabular}{|c|c|c|}
\hline GRAU DE SATISFAÇÃO GERAL & MÉDIA & DESVIO-PADRÃO \\
\hline 1. Totalmente insatisfeito / 7. Totalmente satisfeito & 6,45 & 0,79 \\
\hline
\end{tabular}

\section{CONSIDERAC̣ÕES FINAIS}

Os resultados da pesquisa ilustram que tão importante quanto identificar as imagens de serviços em relação a um público-alvo específico, deve-se entender como as imagens estão configuradas. A partir da revisão da literatura, observou-se que a imagem de serviços é um conjunto de representações, impressões, convicções e redes de significados de um objeto (serviço) armazenado na memória de forma holística, podendo ser configurada a partir de um conjunto de elementos funcionais, simbólicos, cognitivos e emocionais, organizados em torno de alguns elementos centrais e/ou periféricos.

Neste sentido, a pesquisa com os três tipos de paciente possibilitou, além da representação das imagens desses pacientes, verificar a forma como estão organizadas quanto aos seus elementos constituintes, bem como sua organização quanto ao núcleo central. A percepção dos pacientes quanto aos serviços de fisioterapia do Plano de Saúde indica a aplicabilidade da Escala de Diferencial Semântico no contexto de mensuração das imagens.

A identificação dos atributos e seus elementos constituintes (Quadro 4) revelam que as imagens estão muito relacionadas às expectativas e características de cada tipo de paciente. Apesar de haver uma predominância dos elementos cognitivos nos três tipos de paciente, observa-se que, para os neurológicos, há uma primazia dos elementos emocionais e simbólicos. Informações deste tipo são importantes para a gestão dos serviços, pois para cada público podem ser direcionadas ações de acordo com suas expectativas e percepções.

Com base na Teoria do Núcleo Central, foi possível identificar como os usuários dos serviços de fisioterapia organizam suas imagens em torno de seus elementos centrais, intermediários e periféricos. Assim, de acordo com os pacientes entrevistados, a fisioterapia "é um exercício que auxilia na recuperação" (Quadro 5). A compreensão das imagens centrais é um importante instrumento de monitoramento das imagens e mensuração dos conceitos. Como as imagens são um forte influenciador do comportamento dos indivíduos, a compreensão da organização dos respectivos atributos associados à configuração de imagens proporciona um melhor direcionamento das ações na tentativa de aproximar ou afastar os atributos desejados ou não pelos prestadores de serviço.

Os resultados obtidos com a Escala de Diferencial Semântico indicam que o instrumento proposto é adequado e complementa o método adotado para o estudo da configuração e organização de imagens, se transformando em uma importante ferramenta para identificar a percepção dos respondentes em relação ao serviço investigado.

Portanto, o trabalho apresentou duas formas distintas, porém, complementares, para mensurar as imagens de serviços e sua organização na mente do seu público-alvo. As técnicas utilizadas, análise de conteúdo de evocações e Escala de Diferencial Semântico, revelam que ambas são aplicáveis ao contexto de serviços. Entretanto, o que pode direcionar a utilização de uma ou outra técnica, ou ambas, é o problema e os objetivos da pesquisa.

Apesar da contribuição deste trabalho para a configuração de imagens de serviços, ele apresenta limitações. Embora o estudo seja predominantemente exploratório, a amostra utilizada deveria apresentar maior precisão estatística para uma efetiva confirmação e validação, tanto da Escala de Diferencial Semântico, quanto dos resultados provenientes da questão sobre a satisfação geral dos usuários.

\section{Artigo recebido em 23/07/2004 Aprovado para publicação em 25/10/2004}

\section{- Referências Bibliográficas}

ABRIC, J.C. A theoretical and experimental approach to the study of social representations in a situation of interaction. In: FARR, R.; MOSCOVICI, S. (Eds.). Social representations. Cambridge, University Press, p. 169-183, 1984.

ABRIC, J.C. Central system, peripheral system: their functions and roles in the dynamics of social representations. Papers on Social Representations, v. 2, p. 75-78, 1993.
ADLER, S.S.; BUCK, M.; BECKERS, D. PNF: facilitação neuromuscular proprioceptiva. São Paulo: Manole, 1999.

ALBRECHT, K.; ZEMKE, R. Service America! doing business in the new economy. New York: Warner Books Inc., 1990.
ANDERSON, E.W.; FORNELL, C.A customer satisfaction research prospectus. In: RUST, R. T.; OLIVER, R. L. (Eds.). Service quality: new directions in theory and practice. Thousand Oaks: Sage Publications, p. 241-268, 1994.

ANDERSON, E.W.; FORNELL, C. LEHMANN, D.R. Perceived quality, customer satisfaction, market share, and profitability. Working Paper, National Quality Research Center: The University of Michigan, 1992.
BARDIN, L. Análise de conteúdo. Lisboa: Edições 70, 1979.

BARICH, H.; KOTLER, P. A framework for marketing image management. Sloan Management Review, v. 32, n. 2, p. 97104, 1991.

BATESON, J.E.G.; HOFFMAN, K.D. Marketing de serviços. 4. ed. Porto Alegre: Bookman, 2001. 


\section{- Referências Bibliográficas}

BERRY, L.L. Service marketing is different. Business, v. 30, p. 24-29, 1980.

BUSINESS MONITOR INTERNATIONAL. Quarterly Forecast Report - Q3. London, 2000.

CROSBY, P.B. Quality is free: the art of making quality certain. New York: New American Library, 1979.

DE TONI, D.; SCHULER, M. The strategic management of products images: a mensuration of wine images. BALAS 2003 - Business Association of Latin American Studies, São Paulo, 2003.

DEMING, W. E. Out of the crisis. Cambridge: MIT Center for Advanced Engineering Study, 1986.

DICHTER, E. What's in an image. Journal of Consumer Marketing, v. 2, n. 1 , p. $75-81,1985$.

DOBNI, D.; ZINKHAN, G. M. In search of grand image: a foundation analysis. Advances in Consumer Research, v. 17 n.1, p. 110-119, 1990.

FORNELL, C. National and corporate customer satisfaction indexes. A presentation at the World Quality Day. Amsterdam: World Trade Center, Nov. 1991.

FORNELL, C. Productivity, quality, and customer satisfaction as strategic success indicators at firm and national level. Advances in Strategic Management, v. 11A, p. 217-229, 1995.

GARBARINO, E.; JOHNSON, M.S. The different roles of satisfaction, trust, and commitment in customer relationships. Journal of Marketing, v. 63, n. 4, p. 70-87, 1999.
GOLEMAN, D. Inteligência emocional: a teoria revolucionária que redefine o que é ser inteligente. Rio de Janeiro: Objetiva, 1995.

GRÖNROOS, C. Service management and marketing: managing the moment of truth in service competition. Lexington: Free Press, Lexington Books, 1990.

HUFF, L.; FORNELL, C.; ANDERSON, E. W. Quality and productivity: contradictory and complementary. Working Paper, National Quality Research Center: The University of Michigan, 1994.

JOHNSON, M.D.; FORNELL, C.A framework for comparing customer satisfaction across individuals and product categories. Journal of Economic Psychology, v. 12, p. 267-286, 1991.

JURAN, J. M. Juran on leadership for quality. New York: Free Press, 1989.

KOSSLYN, S. M. Image and brain: the resolution of the imagery debate. Cambridge, Massachusetts: The MIT Press, 1996.

LEDOUX, J. O cérebro emocional: os misteriosos alicerces da vida emocional. Rio de Janeiro: Objetiva, 2001.

LEVY, S. J. Symbols for sale. Harvard Business Review, v. 37, n. 4, p. 117-129, July 1959.

LEVY, S. J. Interpreting consumer mythology: a structural approach to consumer behavior. Journal of Marketing, v. 45, n. 3, p. 49-61, 1981.

LINDQUIST, J. D. Meaning of image: a survey of empirical and hypothetical evidence. Journal of Retailing, v. 50, n. 4, p. 29-39, 1975
LOVELOCK, C.; WRIGHT, L. Serviços: marketing e gestão. São Paulo: Saraiva, 2001

MALHOTRA, N. K. Pesquisa de marketing: uma orientação aplicada. 3.ed. Porto Alegre: Bookman, 2001.

MARTINEAU, P. The personality of the retail store. Harvard Business Review, p.47-55, 1958.

MAY, E. G. Practical applications of recent retail image research. Journal of Retailing, v.50, n.4, p.15-20, 1974.

MOSCOVICI, S. A representação social da pscicanálise. Rio de Janeiro: Zahar, 1978.

OLIVER, R. L. Satisfaction: a behavioral perspective on the consumer. New York: Irwin/McGraw-Hill, 1997.

PARAMESWARAN, R.; PISHARODI, R. M. Facets of country of origin image: an empirical assessment. Journal of Advertising, v.23, n.1, p.43-56, 1994.

POIESZ, B. C. The image concept: its place in consumer psychology. Journal of Economic Psychology, v. 10, n. 4, p. 457-472, 1989.

QUEZADA, M. El mensaje medio a medio: qué encierran los diarios, la radio y la T.V. Santiago: Editorial Universitaria, 1992.

REYNOLDS, T. J., GUTMAN J. Advertising is image management. Journal of Advertising Research, v. 24, n. 1, p. 21-37, 1984

SÁ, C. P. de. Sobre o núcleo central das representações sociais. Rio de Janeiro: Vozes, 1996.

SÁ, C. P. de. A construção do objeto de pesquisa em representações sociais. Rio de Janeiro: EdUERJ, 1998.
SCHULER, M. Administração da imagem organizacional: um método de configuração da imagem organizacional. Porto Alegre: PPGA/UFRGS, 2000.

STARKEY, C. Recursos fisioterapêuticos em fisioterapia. 2. ed. São Paulo: Manole, 2001

STERN, B.; ZINKHAN, G. M.; JAJU, A. Marketing images: construct definition, measurement issue, and theory development. Marketing Theory, v. 1, n. 2, p. 201-224, 2001.

THOMSON, A.; SKINNER, A.; PIERCY, J. Fisioterapia de tidy. 12 ed. São Paulo: Santos, 2002.

VERGÈS, P. L'evocation de l'argent: une méthode pour la definition du noyau central d'une representation. Bulletin de Psychologie, v. 45, n. 405 p. 203-209, 1992.

ZEITHAML, V. A. Consumer perceptions of price, quality and value: a meansend model of synthesis of evidence. Journal of Marketing, v. 52, n. 3, p. 2-22, 1988.

ZEITHAML, V. A.; BERRY, L. L.; PARASURAMAN, A. Communication and control processes in the delivery of service quality. Journal of Marketing, v. 52 , n. 2 , p. $35-48,1988$.

ZEITHAML, V. A.; BITNER, M. J. Services marketing: integrating customer focus across the firm. $2^{\text {nd }}$ edition. New York: McGraw-Hill, 2000.

ZEITHAML, V. A.; PARASURAMAN A. BERRY, L. L. Problems and strategies in service marketing. Journal of Marketing, v. 49, n. 2 , p. $33-46,1985$

\section{- Sobre os autores}

\section{Gabriel Sperandio Milan}

LOPP/PPGEP - Escola de Engenharia da UFRGS

Aluno de Doutorado

Endereço: Rua Santo Ceroni, 160, ap. 502 - 95082-230 - Caxias do Sul - RS.

Tel.: (54) 222-2812

E-mail: gabmilan@terra.com.br

\section{Deonir De Toni}

PPGA - Escola de Administração da UFRGS

Aluno de Doutorado

Endereço: Rua Amadeu Zambon, 262 - 95700-230 - Bento Gonçalves - RS.

Tel.: (54) 451-3260

E-mail: deonirdt@terra.com.br

\section{Lisandra Barazetti}

Psicóloga Organizacional

Endereço: Rua Alexandre Fleming, 454 - 95041-520 - Caxias do Sul - RS.

Tel.: (54) 220-8355

E-mail: barazetti@terra.com.br 